\title{
The Influence of Microstructure and Monomer Properties on the Erosion Mechanism of a Class of Polyanhydrides*
}

\author{
ACHIM GÖPFERICH and ROBERT LANGER' \\ Massachusetts Institute of Technology, Department of Chemical Engineering, \\ Building E25, Room 342, Cambridge, Massachusetts 02139
}

\begin{abstract}
SYNOPSIS
The erosion of three different polyanhydrides consisting of sebacic acid (SA) and 1,3bis ( $p$-carboxyphenoxy) propane (CPP) was investigated. Melt cast polymer matrices were prepared from the homopolymer $p$ (SA) and two copolymers, $p$ (CPP-SA) $20: 80$ and $p$ (CPP-SA) $50: 50$. Particular attention was paid to the influence of the polymer matrix microstructure and of the monomers on erosion. Using polarized light microscopy we found that $p$ (SA) and $p$ (CPP-SA) $20: 80$ matrices consist of spherulites. SEM investigations showed that their crystalline parts are more resistant to erosion than their amorphous areas. The matrices erode into highly porous devices, whose porosity is detectable by mercury porosimetry. Using wide-angle $x$-ray diffractometry we found that monomers crystallize inside the pores. DSC investigations showed a maximum of crystallized SA after 2-6 days and a continuous increase of CPP, which stays in the devices for weeks. We conclude that the microstructure and the monomer properties are the two main factors which determine the erosion of these polymers. The obtained data on changes in porosity, crystallinity, polymer matrix thickness, erosion front velocities, crystalline monomer content, and monomer release provides the basis for quantitatively describing the erosion process. (C) 1993 John Wiley \& Sons, Inc.
\end{abstract}

Keywords: polymer $\bullet$ polyanhydride $\bullet$ microstructure $\bullet$ erosion $\bullet$ mechanism $\bullet$ crystallinity

\section{INTRODUCTION}

In recent years the application of degradable polymers has increased rapidly. Controlled drug delivery, and several other areas (e.g., bone implants ${ }^{1}$ or cell transplantation devices ${ }^{2}$ ) have profited from the idea of polymeric materials degrading under the influence of body fluids. In drug delivery there have been significant efforts to optimize the release of drugs from such polymers. Usually investigators focused their interest on the adjustment of release rates. This is very important for the development of new dosage forms and improved medical therapy. However, in some cases progress in controlled drug delivery was not accompanied by a better under-

* Dedicated to Dr. Ruth Dillmann on the occasion of her 60th birthday.

t To whom correspondence should be addressed.

Journal of Polymer Science: Part A: Polymer Chemistry, Vol. 31, 2445-2458 (1993) (C) 1993 John Wiley \& Sons, Inc. CCC 0887-624X/93/102445-14 standing of the polymer properties that control erosion. For many polymers the investigation of erosion has still not shown satisfying results. One of the reasons for this is the complexity of the erosion process.

The erosion of the polymer bulk can be affected by a variety of parameters. Most important is the chemical degradation of bonds in the polymer chains. The velocity of degradation depends on the type of bonds between monomers, ${ }^{3}$ the mobility of water in the polymer, ${ }^{4}$ and on other polymer properties, such as crystallinity. ${ }^{5}$ The solution $\mathrm{pH}$ is a very important external factor, as it has a catalytic effect on the hydrolysis of bonds ${ }^{6}$ and on dissolution rates of the degradation products. During degradation new molecules are created which can comprise monomers as well as oligomers. These substances have properties which are different from each other and from the parent polymer. With their carboxylic groups they can be expected to change the $\mathrm{pH}$ inside cracks and pores and may thus have some feedback 
effect on degradation and erosion. ${ }^{7}$ Many polymers are partially crystalline. The arrangement of crystalline regions may also influence erosion as amorphous regions have been proposed to erode more quickly than crystalline ones. ${ }^{8}$ The erosion mechanism is thus a very specific property of a polymer matrix which even changes for a series of related copolymers.

In this study we elucidate the influence of microstructure and monomer properties on the erosion of a class of related polyanhydrides. We chose one homo- and two copolymers consisting of sebacic acid (SA) and 1,3-bis ( $p$-carboxyphenoxy) propane (CPP) which are shown in Figure 1. The large number of factors which influence erosion demanded a careful choice of analytical methods to investigate the process. We chose methods based on previous results ${ }^{10,11}$ and with the intention to obtain quantitative data which could permit the development of mathematical models to describe the erosion of these polymers in future studies. The morphological characterization of the undegraded polymers ${ }^{10}$ revealed that they are partially crystalline. We decided, therefore, to apply polarized light microscopy to reveal the crystalline microstructure prior to erosion and SEM to detect changes during erosion. The dependence of the solubility of the monomers on $\mathrm{pH}$ was investigated to estimate solubility from $\mathrm{pH}$ measurements during erosion experiments. As previous studies suggested that this class of polymers turns into porous structures ${ }^{11}$ we decided to employ mercury porosimetry to estimate parameters like porosity or mean pore size. By wide-angle $x$-ray diffraction and DSC we investigated whether the monomers are soluble inside the pores. Changes in the thickness of the polymer matrices were measured by light microscopy whereas the surface was investigated by scanning confocal microscopy. Each of these methods alone gives only partial and limited information on the total erosion process. In combination, however, they complement each other and yield detailed information on the process.

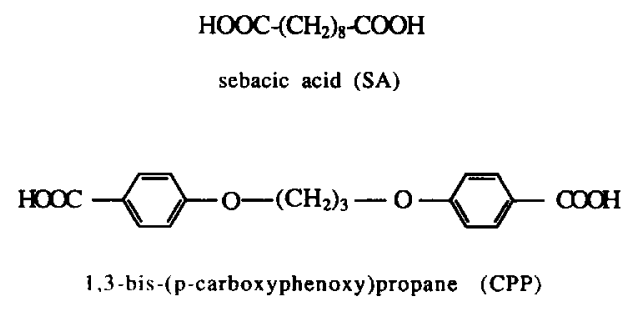

Figure 1. Chemical structure of SA and CPP monomer.

\section{MATERIALS AND METHODS}

\section{Materials}

$p$ (SA) homopolymer (MW 60,000), $p$ (CPP-SA) $20: 80$ (MW 70,000), and $p$ (CPP-SA) $50: 50$ (MW 40,000 ) copolymer, prepared by melt-polycondensation, ${ }^{12}$ as well as SA and CPP monomer, were all obtained from Nova Pharmaceuticals, Baltimore, $\mathrm{MD}$. As a pH-sensitive fluorescent dye, fluorescein5-(and 6) -sulfonic acid was purchased from Molecular Probes Inc., Eugene, OR.

\section{Methods}

\section{Preparation of Polymer Discs and Erosion}

For the investigation of polymer erosion, discs of $1.4 \mathrm{~mm}$ diameter and $1 \mathrm{~mm}$ thickness were prepared by a melt casting method. ${ }^{9}$ They were submitted to erosion in $10 \mathrm{~mL}$ phosphate buffer at $\mathrm{pH} 7.4$ and $37^{\circ} \mathrm{C}$ under gentle shaking ( $60 \mathrm{rpm}$ ). The buffer was changed daily and the $\mathrm{pH}$ measured. Samples were removed until the devices were no longer mechanically stable. They were dried for $24 \mathrm{~h}$ in a $100 \mathrm{~mm}$ $\mathrm{Hg}$ vacuum over phosphorous pentoxide and stored in a desiccator under the same conditions. Weight loss was determined in the dried state.

\section{Polarized Light Microscopy}

For characterization of undegraded polymers, thin films were prepared by melting the polymers between glass slides. The films were examined using a Standard 18 Microscope and taking photographs with an Aufsetzkamera M63 (both from Zeiss, Oberkochen, Germany).

\section{Surface Analysis by Scanning Confocal Microscopy}

The surface of eroded polymer discs was investigated by scanning confocal microscopy using an MRC 500 imaging system from Bio Rad, Hercules, CA. Fluorescein-5- (and 6) -sulfonic acid was added as a fluorescent dye to the buffer medium $(1 \mathrm{mg} / \mathrm{mL})$. For the acquisition of images, the chromophore was excited at $488 \mathrm{~nm}$ and emission was measured at 515 $\mathrm{nm}$. Images from the surface were obtained by averaging a series of 10 pictures differing by $0.1 \mu \mathrm{m}$ in their $z$-position. Pictures from cross sections of eroded discs were taken in the same way with $\Delta z$ $=20 \mu \mathrm{m}$.

The surface $\mathrm{pH}$ was measured by taking advantage of the $\mathrm{pH}$ dependence of the fluorescein-5-(and 
6) -sulfonic acid emission spectrum. First the system was calibrated with dye solutions of different $\mathrm{pH}$. Pictures were simultaneously taken at 540 and 600 $\mathrm{nm}$. The average gray level ratio was calculated for each $\mathrm{pH}$, which provided a calibration curve. Eroded polymer discs were placed into the well of a Boerner slide covered with phosphate buffer $\mathrm{pH}=7.4 \mathrm{con}$ taining $1 \mathrm{mg} / \mathrm{mL}$ dye and covered with a glass cover slip. Optical cross sections of the buffer were taken starting $200 \mu \mathrm{m}$ above the polymer surface and proceeding in steps of $1 \mu \mathrm{m}$ towards the polymer disc. Pictures were again taken at 540 and $600 \mathrm{~nm}$. Dividing the corresponding gray levels obtained in the two channels it was possible to calculate the $\mathrm{pH}$ from the calibration curve. The two-dimensional pH profile was further simplified as the $\mathrm{pH}$ parallel to the polymer surface does not change. The resulting onedimensional average profile was calculated from 384 individual profiles. The software for all calculations was written on an IBM compatible PC in Turbo Pascal (Borland International Inc., Scotts Valley, CA).

\section{Microstructural Investigations by Scanning Electron Microscopy (SEM)}

For the SEM studies the dried samples were fixed in a split specimen mount from Energy Beam Inc. They were brittle enough so that the upper part of the sample could be broken off with tweezers. Images were obtained from gold-coated samples with a Stereoscan 250 MK3 from Cambridge Instruments.

\section{Determination of Monomer Solubility}

For solubility determination a surplus of monomer was dispersed in phosphate buffer and stored at $37^{\circ} \mathrm{C}$ for $48 \mathrm{~h}$. Different $\mathrm{pH}$ values were adjusted by adding $0.1 M \mathrm{NaOH}$ or $0.1 \mathrm{M} \mathrm{HCl}$. Prior to analysis the samples were filtered through $0.45 \mu \mathrm{m}$ pore size filters (Millex- $\mathrm{AP}_{4}$ from Millipore, Bedford, MA).

\section{Investigation of the Crystallinity by Wide-Angle $X$-Ray Diffraction and Differential Scanning Calorimetry (DSC)}

Wide-angle $x$-ray diffraction spectra were taken with a Rigaku Rotaflex Diffractometer from Rigaku Corp. (Danvers, MA) with $S=0.05^{\circ}$ and $T=5 \mathrm{~s}$ using a Nickel-filtered $\operatorname{CuK} \alpha$ source. The data were assembled and evaluated on a Micro Vax II computer. For the determination of crystallinities by DSC a 7 Series Thermal Analysis System from Perkin-Elmer (Newton Center, MA) was used. The thermograms were recorded with a heating rate of $10^{\circ} \mathrm{C} / \mathrm{min}$.

\section{Structural Investigations Using Light Microscopy}

The movement of the erosion front was followed by investigating cross sections of dried samples with a zoom macroscope (Model M420, Wild Heerbrugg, Heerbrugg, Switzerland).

\section{Determination of Porosity by Mercury Porosimetry}

The porosity of eroded samples was determined by mercury porosimetry using a Poresizer 9320 ( Micrometrics, Norcross, GA). The measurements were performed in a range from 0.5 to 30000 psi using a penetrometer with a $5 \mathrm{~mL}$ bulb volume (Model 920 61707-00 from Micromeritics).

\section{Determination of Monomer Release by HPLC}

For the determination of SA and CPP concentrations an isocratic HPLC method was developed. The mobile phase was composed of $1200 \mathrm{~mL}$ acetonitrile, $1500 \mathrm{~mL}$ water, and $100 \mathrm{~mL} 1 M \mathrm{HCl}$ solution. A PRP-1 Hamilton column $4.1 \times 150 \mathrm{~mm}$ with $5 \mu \mathrm{m}$ particles purchased from Rainin Instruments (Woburn, MA) was used as stationary phase. SA was detected at $210 \mathrm{~nm}$ and CPP at $246 \mathrm{~nm}$. The run time was $10 \mathrm{~min}$ at a flow rate of $0.8 \mathrm{~mL} / \mathrm{min}$. All measurements were performed on a Waters HPLC setup comprising a M510 pump, a M490 UV detector, and a Wisp 712 autosampler, all from Millipore, Bedford, MA.

\section{RESULTS AND DISCUSSION}

\section{Structural Investigation by Polarized Light Microscopy Prior to Erosion}

Under polarized light noneroded homopolymer, $p$ (SA), and noneroded copolymer containing $20 \%$ CPP, $p$ (CPP-SA) $20: 80$, show Maltese crosses [Figs. 2(a) and (b)] which are typical of polymers consisting of spherulites. ${ }^{13}$ The Maltese crosses of the anhydrides show circular arranged bands ${ }^{14}$ which are clearly visible [Fig. 2(a)]. These results from the arrangement of the crystalline regions within the spherulites. From etching experiments with spherulitic polymers it is known that they consist of crystalline arrays of branching units which spread radially from the center of the structure and are embedded into amorphous polymer areas. ${ }^{15}$ In the case of banded spherulites these crystalline branches undergo a periodical chain twisting with 

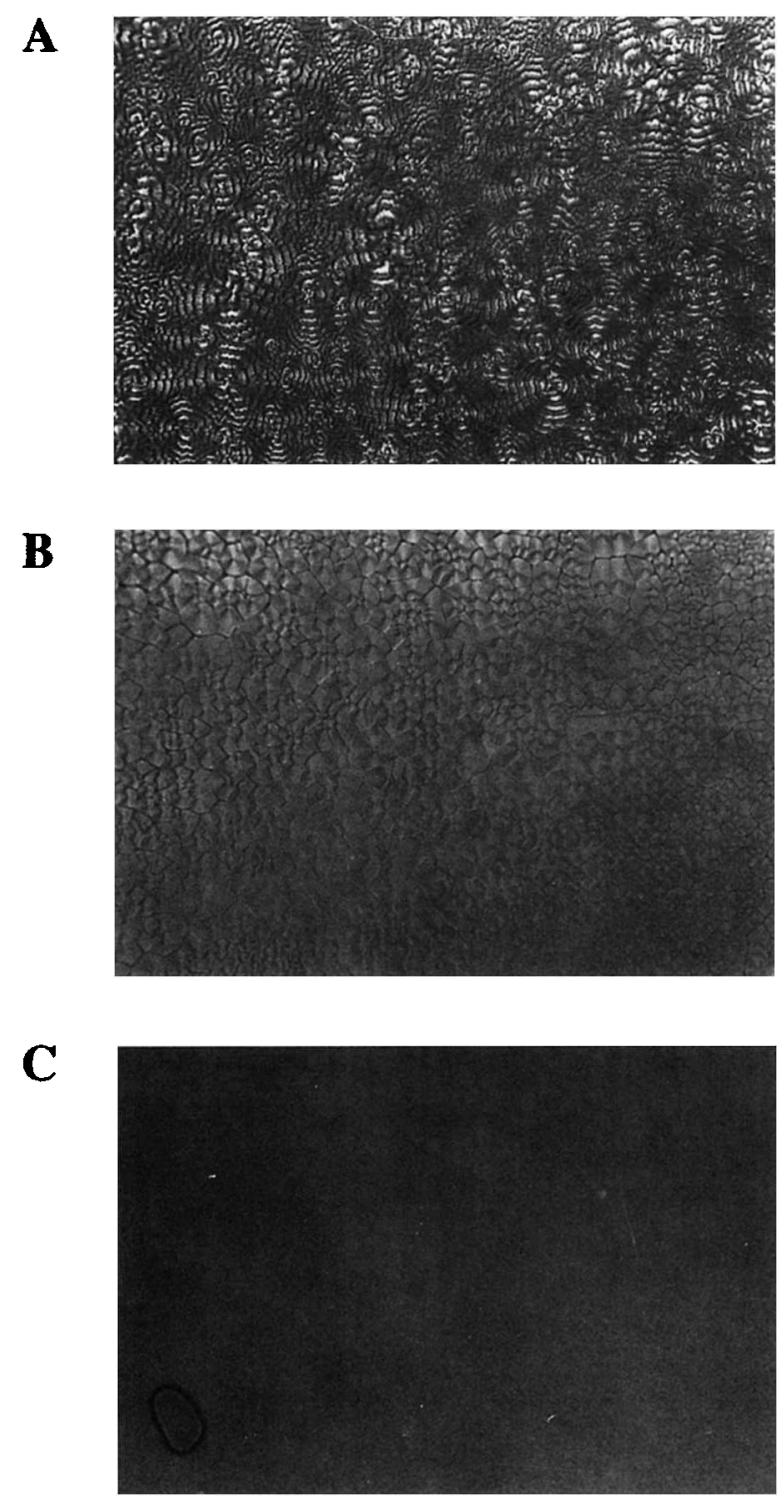

Figure 2. Pictures obtained by polarized light microscopy from thin polymer films $(250 \times):$ (A) $p$ (SA), (B) $p$ (CPP-SA) $20: 80$, (C) $p$ (CPP-SA) $50: 50$.

increasing distance from the origin. The optical axis of the branches is, thereby, periodically rotated causing the banded appearance under crossed polaroids. ${ }^{16}$ With regard to these results this class of polymers has a distinct microstructure which may be of importance for their erosion. In the case of $p$ (CPP-SA) $50: 50$ the equal presence of both monomers leads to an irregular arrangement of the monomers in the polymer chain. ${ }^{10}$ This prevents, to a large extent, the crystalline arrangement of the polymer chains, so that crystalline superstructures cannot be created [Fig. 2(c)].

\section{The Change of Microstructure during Erosion}

From investigations by polarized light microscopy it can be concluded that this class of polyanhydrides has a distinct microstructure for most monomer ratios. We wanted to see how this microstructure changes during erosion. To this end, eroded samples were broken up and the cross sections investigated by SEM. The polymer matrix surface was investigated by scanning confocal microscopy.

After $24 \mathrm{~h}$ of erosion, the polymer surface is covered by a network of cracks. ${ }^{17}$ Figure 3 shows the surface of a $p$ (CPP-SA) $20: 80$ matrix. Besides these changes which affect mainly the surface of the polymer matrix there are major changes inside the polymer which became visible by SEM. Figure 4 (a) shows a cross section through $p$ (CPP-SA) $20: 80$ after 3 days of erosion. As known from previous investigations ${ }^{11}$ there are three different layers: two outer layers where the buffer has already eroded the polymer and an inner layer where the polymer is noneroded. These layers are sharply separated whereby the boundary between them moves from the surface to the center of the discs during erosion. ${ }^{18}$

Figure $4(\mathrm{~b})$ shows this boundary at higher magnification: the left part of the picture shows the noneroded part; the right part the eroded area of the disc. In the noneroded part, spherulites with a diameter of ca. $20 \mu \mathrm{m}$ are visible and show again the circular arrangement of bands. At higher magnification the microstructure of the bands become visible [Fig. $4(\mathrm{c})$ ]. The eroded part of the polymer in contrast has a different appearance. Figure 4 (b) shows that single spherulites are reduced to their dendritic skeleton. The banding has disappeared and their center becomes visible. At higher magnification the fine structure of these eroded spherulites is visible. Figure 4 (d) shows that they consist of loosely associated polymer plates separated by pores of size

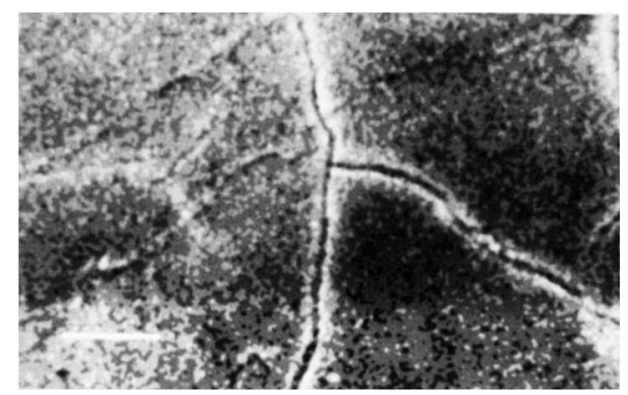

Figure 3. Picture of the $p$ (CPP-SA) $20: 80$ surface after $18.5 \mathrm{~h}$ in phosphate buffer $\mathrm{pH} 7.4$ at $37^{\circ} \mathrm{C}$, taken by scanning confocal microscopy ( scale bar $=100 \mu \mathrm{m}$ ). 

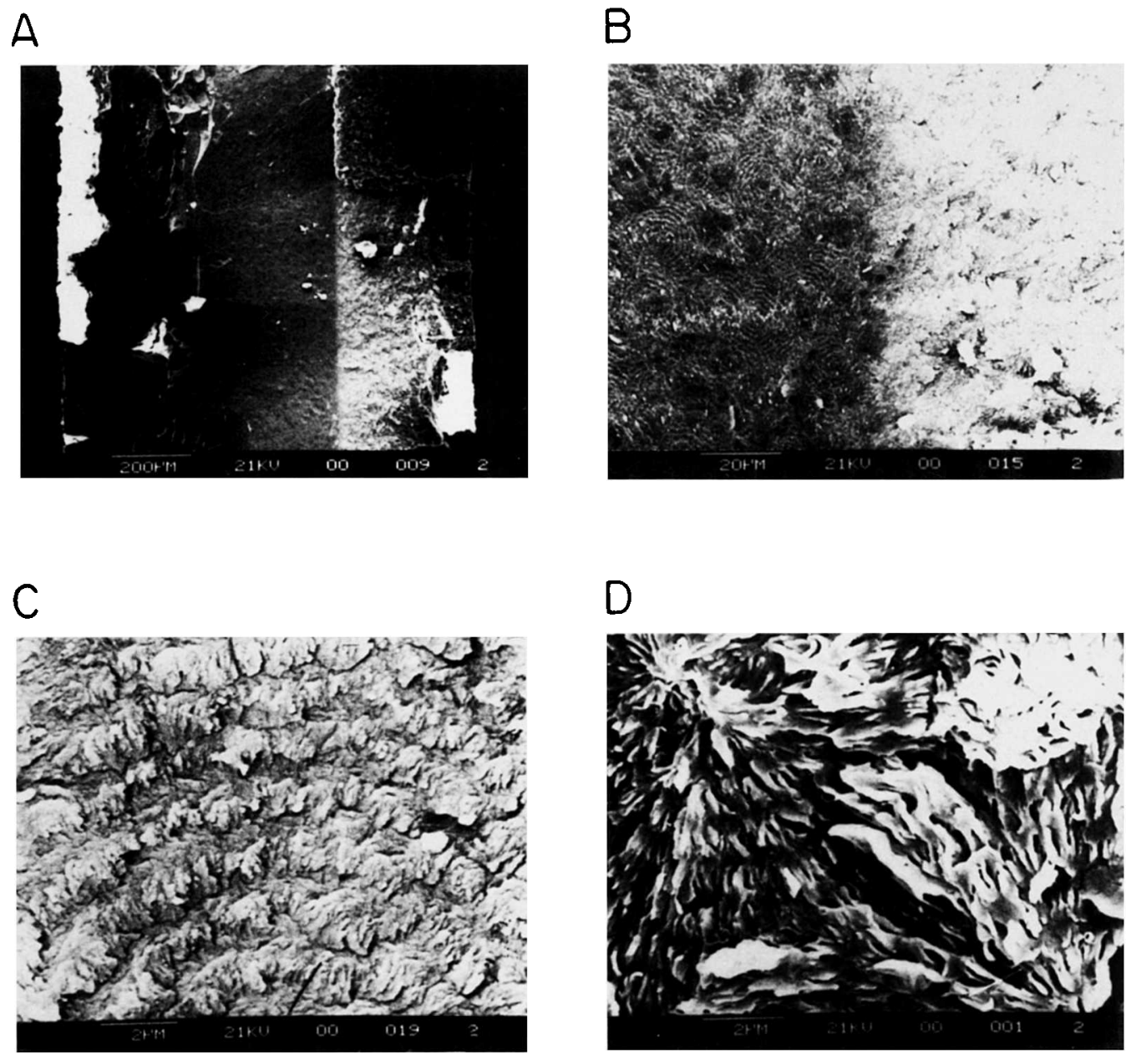

Figure 4. SEM pictures of eroded $p$ (CPP-SA) $20: 80$ discs after 3 days: (A) cross section through the device, (B) erosion front, (C) banding of undegraded spherulite in detail, (D) crystalline skeleton of an eroded spherulite.

in the range of $0.1-2 \mu \mathrm{m}$. It is obvious that the polymer has changed from nonporous bulk to highly porous material whereby amorphous parts degrade faster than crystalline parts. The process is neither bulk nor perfect surface erosion ${ }^{19}$ but rather has elements of both. The moving erosion front is characteristic of surface erosion whereas the remaining porous shell stems from bulk erosion. The same structural changes are observable for $p(\mathrm{SA})$. $p$ (CPP-SA) $50: 50$, in contrast, is too amorphous to build up crystalline superstructures.

\section{The Solubility of the SA and CPP Monomer}

Due to the degradation of polymer chains, large amounts of monomers are released into the pores created during erosion and finally diffuse into the buffer outside the discs. We determined the solu- bility of SA and CPP to make sure that they are sufficiently soluble in the degradation medium outside the polymer matrix. Furthermore we were interested in their $\mathrm{p} K_{a}$ values to determine which is the stronger acid. Figure 5 displays the solubility profiles of the monomers depending on $\mathrm{pH}$. As expected for carboxylic acids the solubility of both compounds can be increased by increasing $\mathrm{pH}$. To obtain a continuous function, the experimental data was smoothed using interpolating splines of third degree ${ }^{20}$ which describe the $\mathrm{pH}$ between experimental values by a third-degree polynomial. By searching for points of inflexion in the polynomials it was possible to estimate the $\mathrm{p} K_{a}$ values of both substances. They were $\mathrm{p} K_{a_{1}}=4.8$ and $\mathrm{p} K_{a_{2}}=5.6$ for SA and $\mathrm{p} K_{a_{1}}=4.5$ and $\mathrm{p} K_{a_{2}}=7.9$ for CPP. The two first $\mathrm{p} K_{a}$ values are in good agreement with data for comparable carboxylic compounds ${ }^{21}$ and show that 


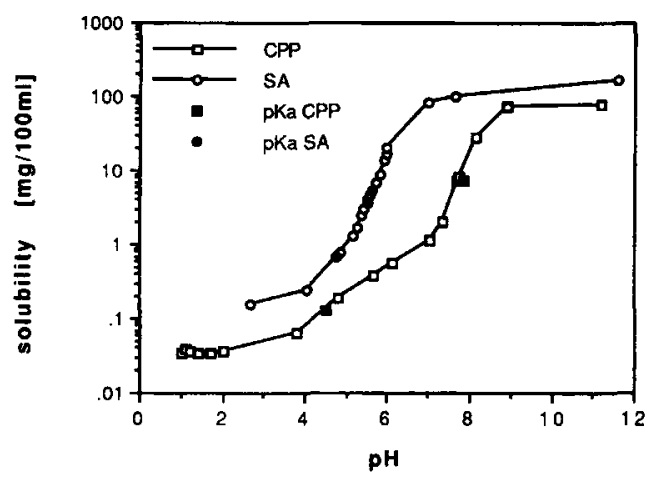

Figure 5. Solubility profiles of CPP and SA.

both monomers are weak acids. The curves demonstrate that SA is at least five times more soluble than $\mathrm{CPP}$ at $\mathrm{pH}$ values below 7.4.

\section{The Change of Buffer $\mathrm{pH}$ and Surface $\mathrm{pH}$ during Erosion}

To ensure the solubility of the released monomers the $\mathrm{pH}$ in the buffer medium was measured whenever it was changed. The $\mathrm{pH}$ time profiles during erosion are shown in Figure 6. All of them show that the $\mathrm{pH}$ is lowered by the release of monomer during erosion. They all have a distinct minimum which marks the time with the highest release rate of monomers. From the solubility profiles in Figure 5 we conclude that the $\mathrm{pH}$ was always high enough to allow the dissolution of all monomers in the vial and to maintain sink conditions.

In the case of $p$ (CPP-SA) $20: 80$ the surface $\mathrm{pH}$ was measured by scanning confocal microscopy. Figure 7 (a) shows the calibration curve which relates the measured ratio of the gray levels from channel $1(540 \mathrm{~nm})$ and channel $2(600 \mathrm{~nm})$ to $\mathrm{pH}$.

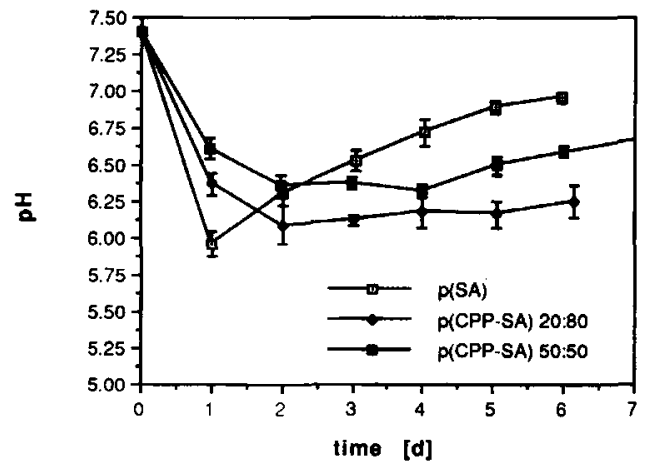

Figure 6. pH in the buffer medium during erosion.
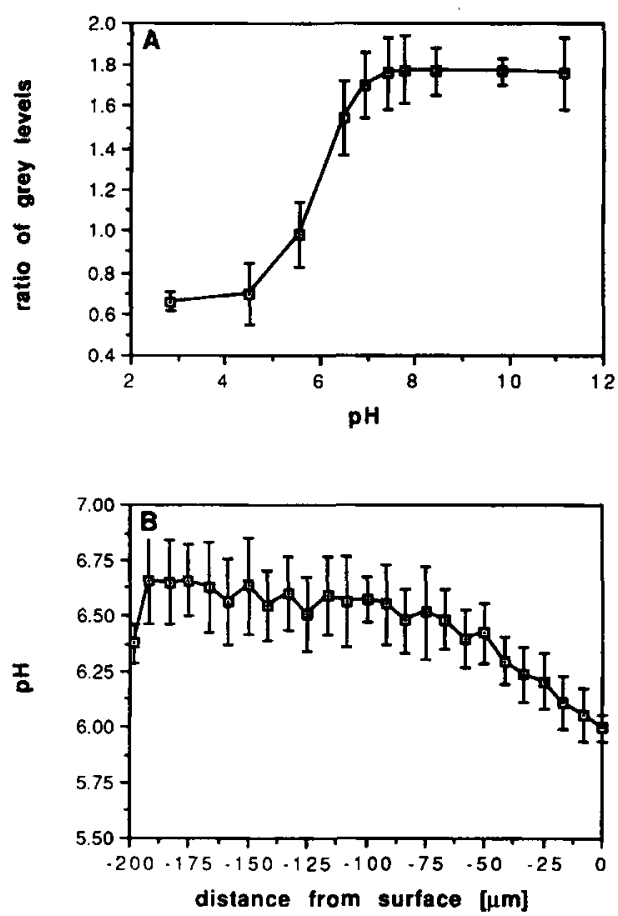

Figure 7. (A) Calibration curve for the determination of the surface $\mathrm{pH}$ by scanning confocal microscopy. Ratio of gray levels in channel $1(540 \mathrm{~nm})$ and channel $2(600$ $\mathrm{nm}$ ). (B) $\mathrm{pH}$ profile of the buffer next to the surface of $p$ (CPP-SA ) $20: 80$ discs after $18.5 \mathrm{~h}$ of erosion $(n=6)$.

Figure 7 (b) shows the pH profile of the buffer solution above the polymer surface. It is seen that the pH drops when approaching the surface. This shows that the $\mathrm{pH}$ in the buffer solution measured with a glass electrode is higher than the value close to the surface.

The $\mathrm{pH}$ inside the polymer has been reported ${ }^{17}$ to be ca. 5 . We obtained the same value after the hydrolysis of the polymer in phosphate buffer under reflux. From these results in combination with results from SEM we conclude that the polymer creates its own $\mathrm{pH}$ micro-climate inside the porous network, which reaches even to the layers close to the surface. The $\mathrm{pH}$ is, thereby, mainly controlled by the two monomers. It can, however, not be excluded that end groups of polymer and oligomer chains contribute to $\mathrm{pH}$.

\section{Changes of the Crystallinity of the Polymers during Erosion}

By polarized light microscopy and SEM it was shown that $p$ (SA) and $p(\mathrm{CPP}-\mathrm{SA}) 20: 80$ have a distinct microstructure which is related to their crystallinity. 
During erosion this microstructure breaks down whereby the crystalline regions exhibit a higher resistance to degradation than amorphous regions, causing changes in polymer matrix crystallinity. The $\mathrm{pH}$ investigations have shown that the $\mathrm{pH}$ inside the porous layers is determined by the monomers which have a limited solubility. In the case that the degradation of the polymer chains is faster than the diffusion of monomers to the matrix surface it could be that the monomers crystallize inside the porous network of the devices. For the investigation of changes in crystallinity during erosion wide-angle $x$-ray diffraction and DSC were used. The former is an excellent method to reveal qualitative changes in crystallinity. The latter is useful for quantitative evaluation. As an example of an x-ray diffraction spectrum, the pattern of noneroded $p$ (CPP-SA) $20: 80$ is shown in Figure 8(c). There are marked peaks at $2 \theta=19.5^{\circ}, 20.8^{\circ}, 23.1^{\circ}$, and $25.3^{\circ}$ which are in good agreement with previous findings. ${ }^{10} \mathrm{Fig}$ ure 9 shows how the diffraction pattern changes during erosion. Already after $24 \mathrm{~h}$ new peaks at $2 \theta$ $=8^{\circ}, 17^{\circ}, 21.2^{\circ}$, and $23.8^{\circ}$ appear in the spectrum and grow with time. The relative intensity of these peaks increases, whereas the relative intensity of the polymer peaks decreases during erosion. This indicates that the erosion of the polymer is accompanied by the growth of new crystal forms which are not present prior to erosion. As large amounts of monomers are set free due to the erosion of the polymer it seemed likely that these peaks originated from monomer crystals. This could easily be proved by comparing the powder diffraction pattern of the monomers shown in Figures $8(\mathrm{a})$ and $(\mathrm{b})$ to those of the eroded polymer discs. It is obvious that the peaks which appear during erosion of the devices originate from the monomers. The peaks at $2 \theta=8^{\circ}$, $21.2^{\circ}$, and $23.8^{\circ}$ in the spectra of eroded discs match the spectrum of $\mathrm{SA}$ whereas the peak at $2 \theta=17^{\circ}$ can be found in the CPP diffraction pattern. From these findings it can be concluded that the monomers crystallize during erosion inside the porous network of the eroded polymer matrix. The anhydride bonds, therefore, degrade whenever exposed to water despite being surrounded by a saturated solution of hydrolysis products. Based on these results the polymers were investigated by DSC. First the monomers were examined to determine their melting point as well as their melting enthalpy. Figures 10 (a) and (b) show the thermograms which are both characterized by a clear melting peak. SA has a melting point of $T_{m_{\mathrm{SA}}}=135.6 \pm 0.4^{\circ} \mathrm{C}$ and $\mathrm{CPP}$ of $T_{m_{\mathrm{CPP}}}=323.8 \pm 1.4^{\circ} \mathrm{C}$. By integration the melting enthalpy was determined to be $\Delta H_{m_{\mathrm{SA}}}=218 \pm 5 \mathrm{~J} /$
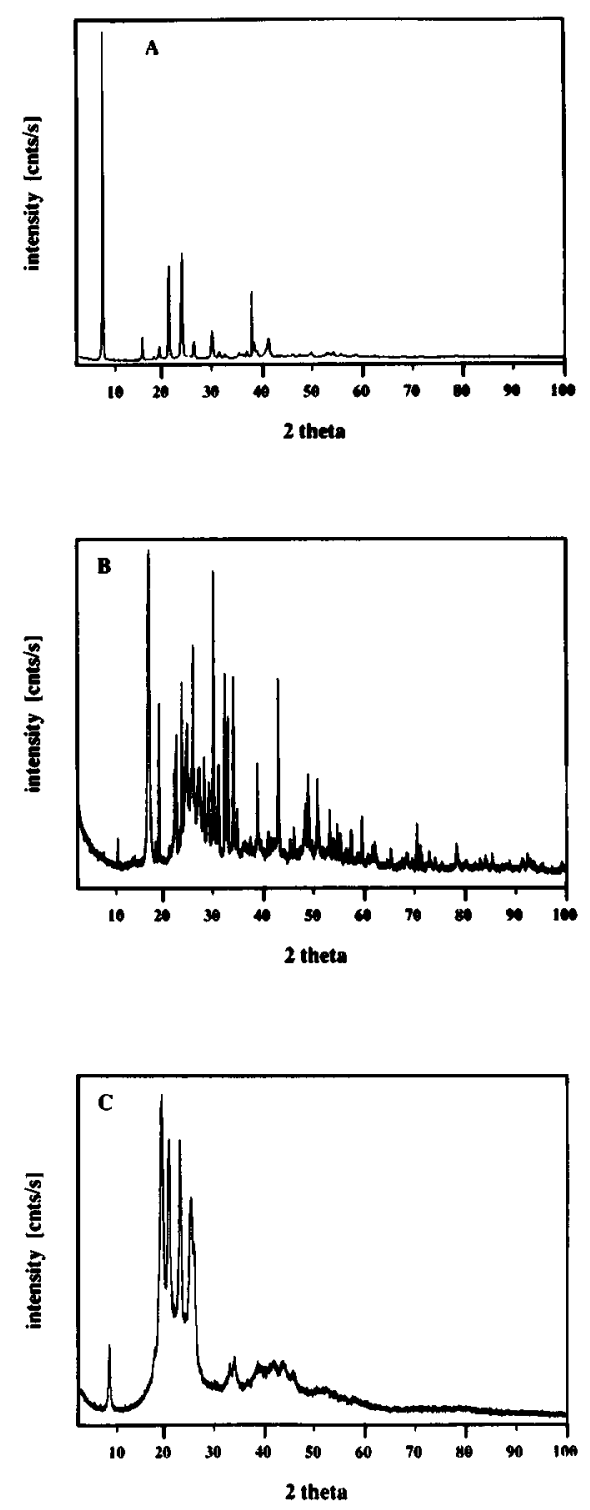

Figure 8. Wide-angle x-ray diffraction spectra: (A) SA monomer, (B) CPP monomer, (C) undegraded $p$ (CPPSA) $20: 80$.

$\mathrm{g}$ for SA and $\Delta H_{m_{\mathrm{CPP}}}=181 \pm 7 \mathrm{~J} / \mathrm{g}$ for CPP. Figures 11 (a) - (c) show the thermograms of the three polymers during erosion. The noneroded $p(\mathrm{SA})$ polymer has one endothermic peak at $T_{m}=81^{\circ} \mathrm{C}$ [ Fig. 11(a)] which is the melting peak of the crystallites. With increasing time a slight shoulder appears in the range $T_{m}=100-110^{\circ} \mathrm{C}$ which, based on the results from wide-angle $x$-ray investigations and thermograms of the monomers, can be identified as crystallized SA. The two copolymers show similar results. The noneroded materials show one endothermic peak at $T_{m}=77^{\circ} \mathrm{C}$ in the case of $p(\mathrm{CPP}-$ 

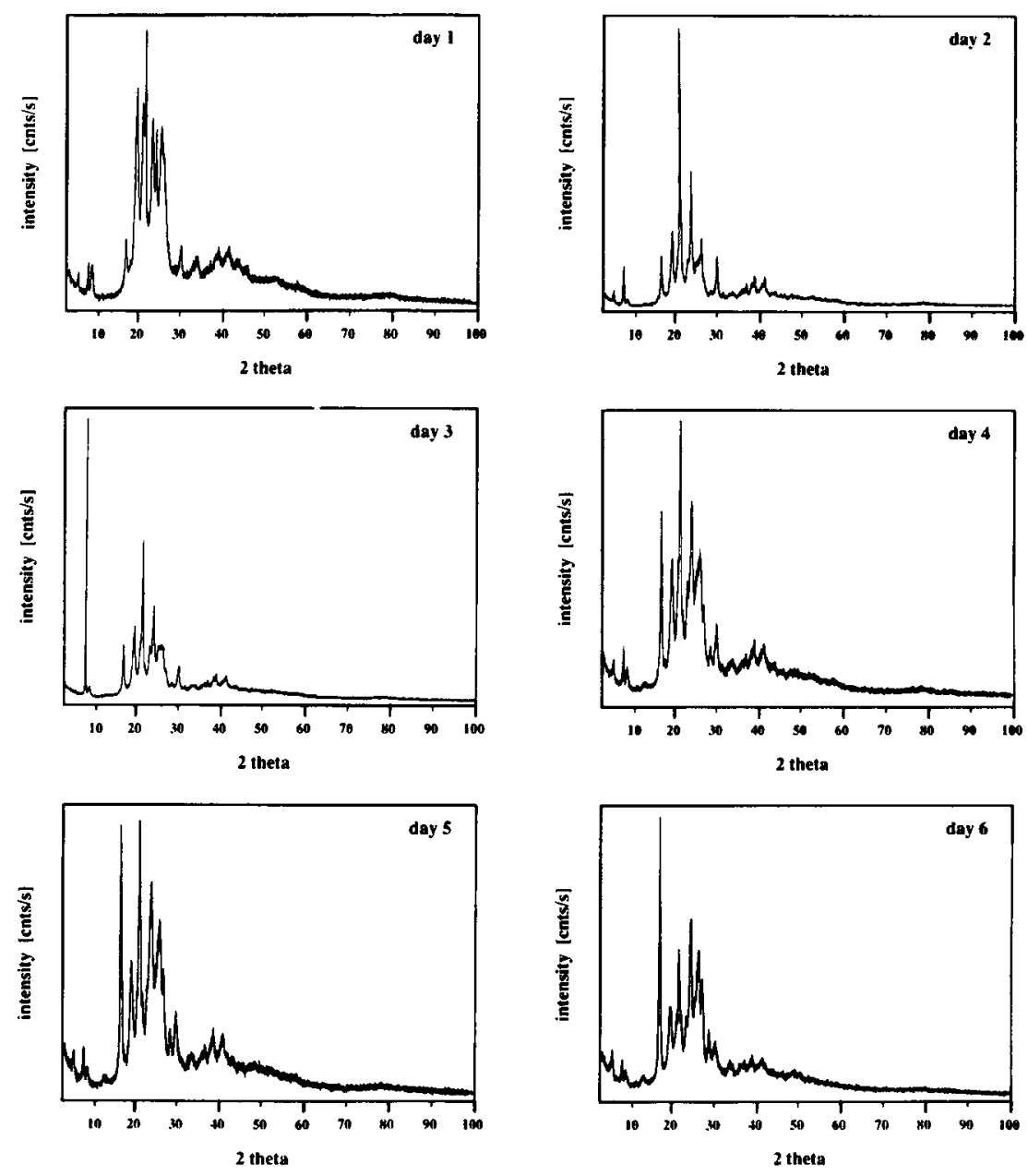

Figure 9. Changes of the wide angle x-ray diffraction spectra of $p$ (CPP-SA) $20: 80$ during erosion.

SA) $20: 80$ [Fig. $11(\mathrm{~b})]$ and at $T_{m}=56^{\circ} \mathrm{C}$ in the case of $p$ (CPP-SA) $50: 50$ [Fig. 11(c)]. Two additional peaks at $T_{m}=100-150^{\circ} \mathrm{C}$ and at $T_{m}=250$ $325^{\circ} \mathrm{C}$ are visible. The former is again caused by crystallized SA monomer; the latter corresponds to crystallized CPP.

For quantitative evaluation of these results, the crystallinity of the devices during erosion was calculated. From DSC measurements of eroded polymer discs, the melting enthalpy was obtained by integration of the melting peaks. The melting enthalpy of the polymer crystallites could be obtained from the literature.$^{10}$ The crystallinity in percent was obtained by dividing the experimental by the tabulated values. The results of these calculations are shown in Figure 12. In the $p$ - (SA) homopolymer the crystallinity increases with time whereas it decreases in the case of the two copolymers. The increasing crys- tallinity in $p$ - (SA) during erosion indicates that the crystalline regions in this polymer are more resistant to erosion than the amorphous parts. ${ }^{8}$ The decreasing crystallinity in the copolymers shows that the crystalline parts of the copolymers degrade substantially faster than those in the homopolymer which is probably due to the disturbance of the crystallites by the increasing presence of CPP monomer.

It was possible to estimate the content of crystallized monomers inside the matrices during erosion based on the melting enthalpy of the pure monomers. The results for $p(\mathrm{SA})$ are shown in Figure 13(a). The content of crystallized SA monomer reaches approximately $7 \%$ of the total disc weight after 1-2 days and drops to zero on the fourth day. The time of the maximum coincides with the $\mathrm{pH}$ minimum of the buffer (Fig. 6). This indicates that the crystallization is a consequence of the large re- 

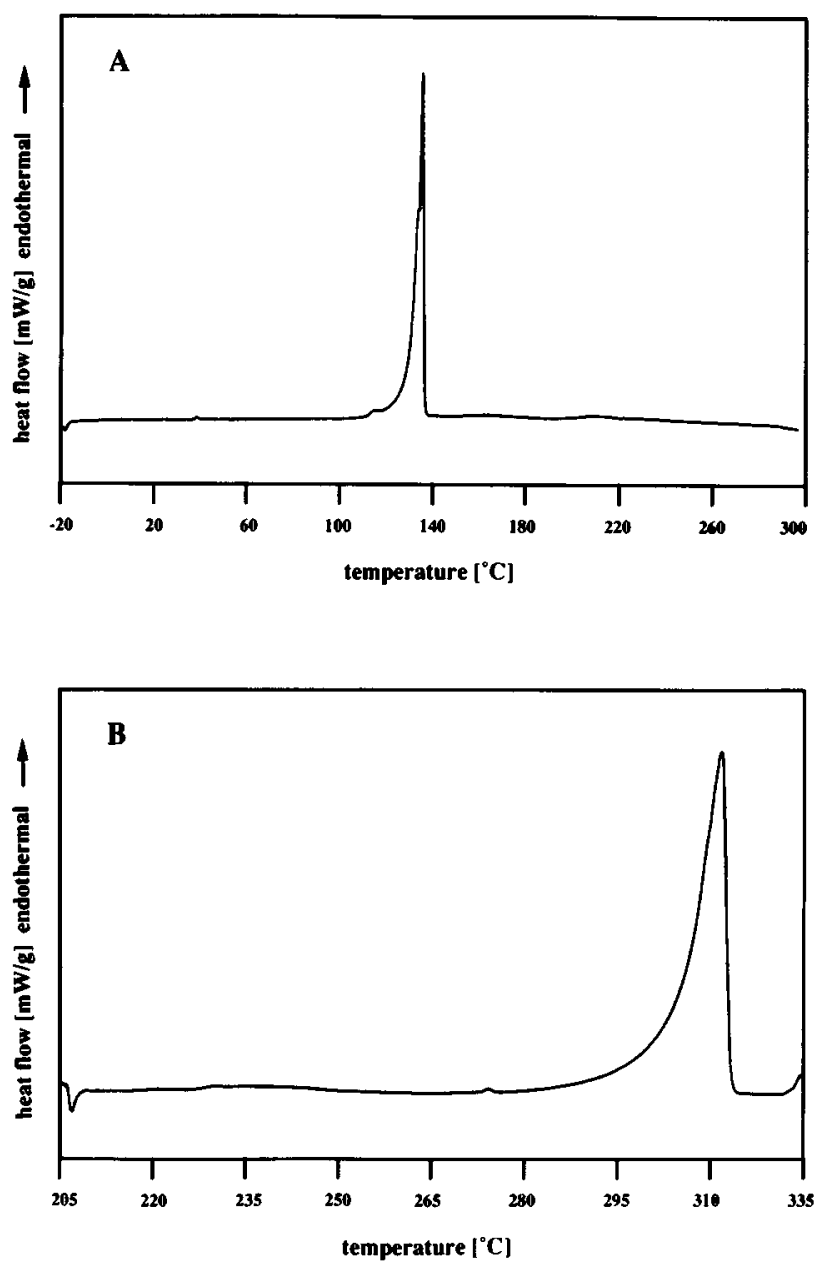

Figure 10. DSC thermograms of the monomers: (A) SA, (B) CPP.

lease of monomers after 1 day. Whenever the release is too slow to keep the buffer inside the pores saturated with monomer, the crystals dissolve again and the mass of crystallized monomer begins to drop. In the case of $p$ (CPP-SA) $20: 80$ the results are similar. Figure 13(b) shows to what extent both monomers crystallize during device erosion. The SA content reaches a maximum which coincides with the pH minimum in the buffer medium (Fig. 6). Compared to SA, the content of crystalline CPP increases linearly with time. Surprisingly, the mass of CPP is equal to or higher than that of SA even though the CPP content is $\frac{1}{5}$ that of SA. This can be explained with the higher solubility of SA compared to CPP, which prevents SA from crystallizing to a larger extent. In the case of $p$ (CPP-SA) 50 : 50 the results are similar to $p$ (CPP-SA) $20: 80$. Figure 13(c) shows that the content of crystalline
SA again reaches a maximum which coincides with the minimum of the $\mathrm{pH}$ in the buffer medium (Fig. 6). The mass of crystallized CPP monomer is higher than in $p$ (CPP-SA) $20: 80$ due to its higher content according to the composition in this copolymer.

The wide angle $\mathrm{x}$-ray measurements and DSC investigations establish that the chemical and physical properties of the polymers changed completely during erosion. Eroded $p(\mathrm{SA})$ consists of the crystalline spherulitic skeleton whereas the copolymers consist of crystallized CPP. It cannot, however, be excluded that small amounts of anhydride oligomers are still present in these polymers which are not detectable by DSC but were seen by more sensitive methods. ${ }^{11}$

\section{The Change of Shape during Erosion}

The results from the previous sections revealed changes in the microstructure as well as the chemical composition of the polymer matrices. We were interested in how the geometry of the devices would be affected by these changes. Of special interest were changes in the total thickness and the movement of the erosion front. By light microscopy we distinguished between the eroded shell and the noneroded inner part of cross sections of polymer discs. Figure 14 shows how the thickness of these two zones in the polymers change with time. In the $p(\mathrm{SA})$ samples no eroded zone was visible. Therefore only the change in total thickness could be measured [Fig. 14 (a) ]. Surprisingly the thickness does not change substantially for several days. After 6 days the polymer discs are too fragile to be removed from the buffer solution without being damaged. During the monomer release studies it could, however, be observed that the total thickness of these devices remains the same for several weeks. In the case of the two copolymers the thickness of the eroded and the noneroded part was measured and added to calculate the total thickness. Figures 14 (b) and (c) show that there is again no substantial change in total thickness. An interesting observation is that the erosion front in both copolymers reaches the center of the discs after nearly the same time. In general, we conclude that matrices from all polymers keep their original shape for a long time even if the erosion front has reached the center of the matrices. This is due to the crystalline polymer structures which are resistant to degradation and the precipitated CPP monomer. In the case of the crystalline structures we assume, based on previous studies, that they consist of a mixture of partially degraded polymer chains which consist mainly of CPP. ${ }^{11}$ 

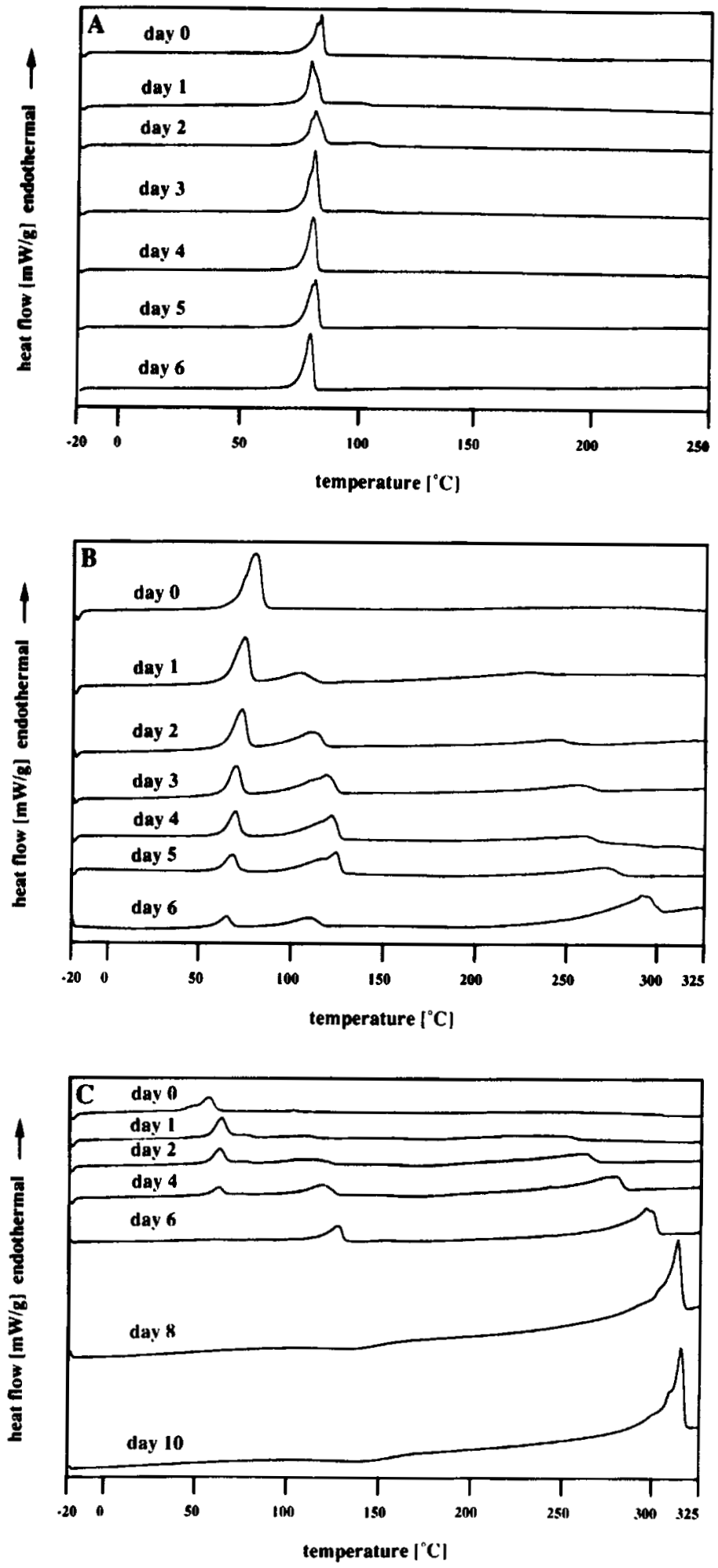

Figure 11. Changing DSC thermograms during erosion: (A) $p$ (SA), (B) $p$ (CPP-SA) $20: 80$, (C) $p$ (CPP-SA) $50: 50$. 


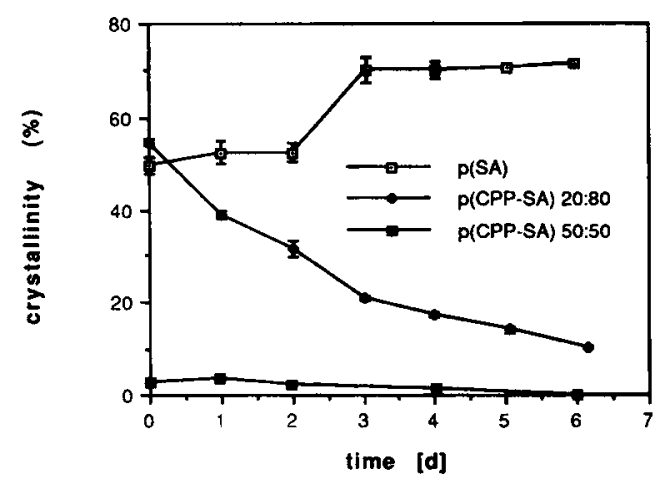

Figure 12. Changes in polymer crystallinity during erosion.

\section{Changes of Matrix Weight and Porosity during Erosion}

As the height and diameter of the polymer discs do not change substantially with time, it was useful to
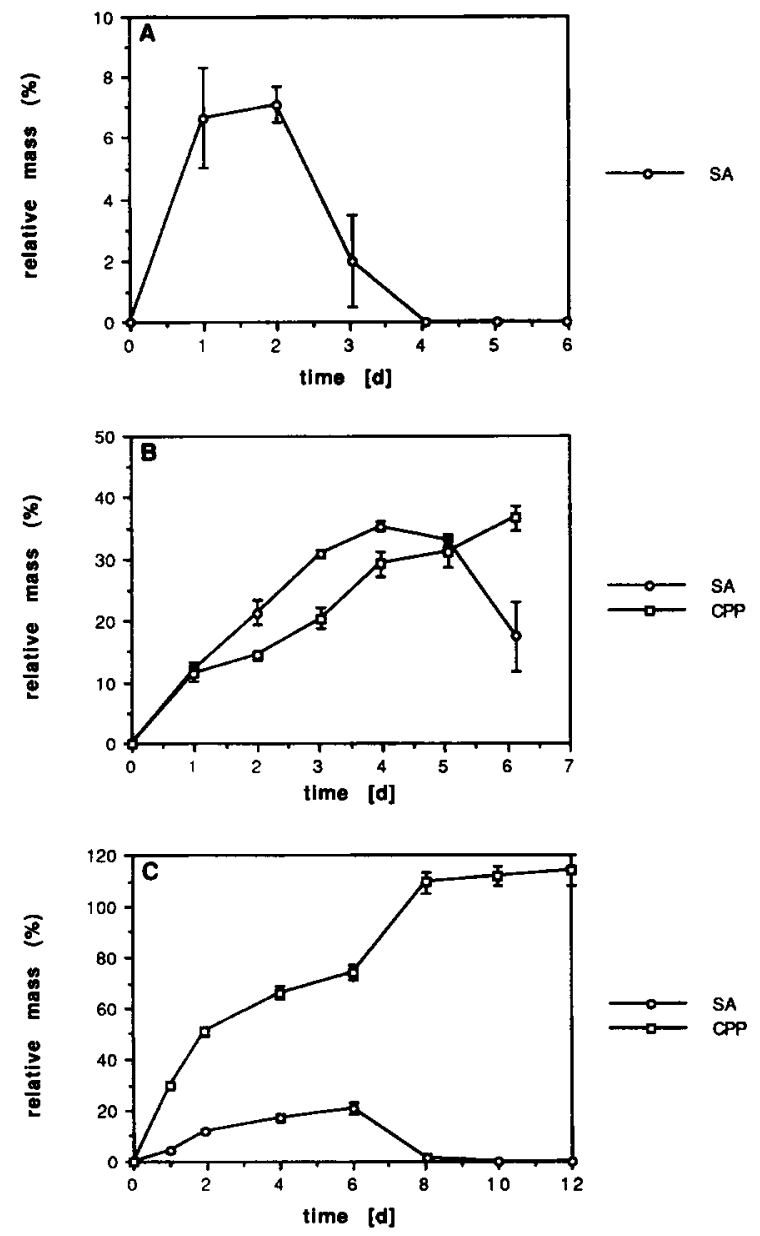

Figure 13. Monomer content of polymer matrices during erosion: (A) $p$ (SA), (B) $p$ (CPP-SA) $20: 80$, (C) $p(\mathrm{CPP}-\mathrm{SA}) 50: 50$.
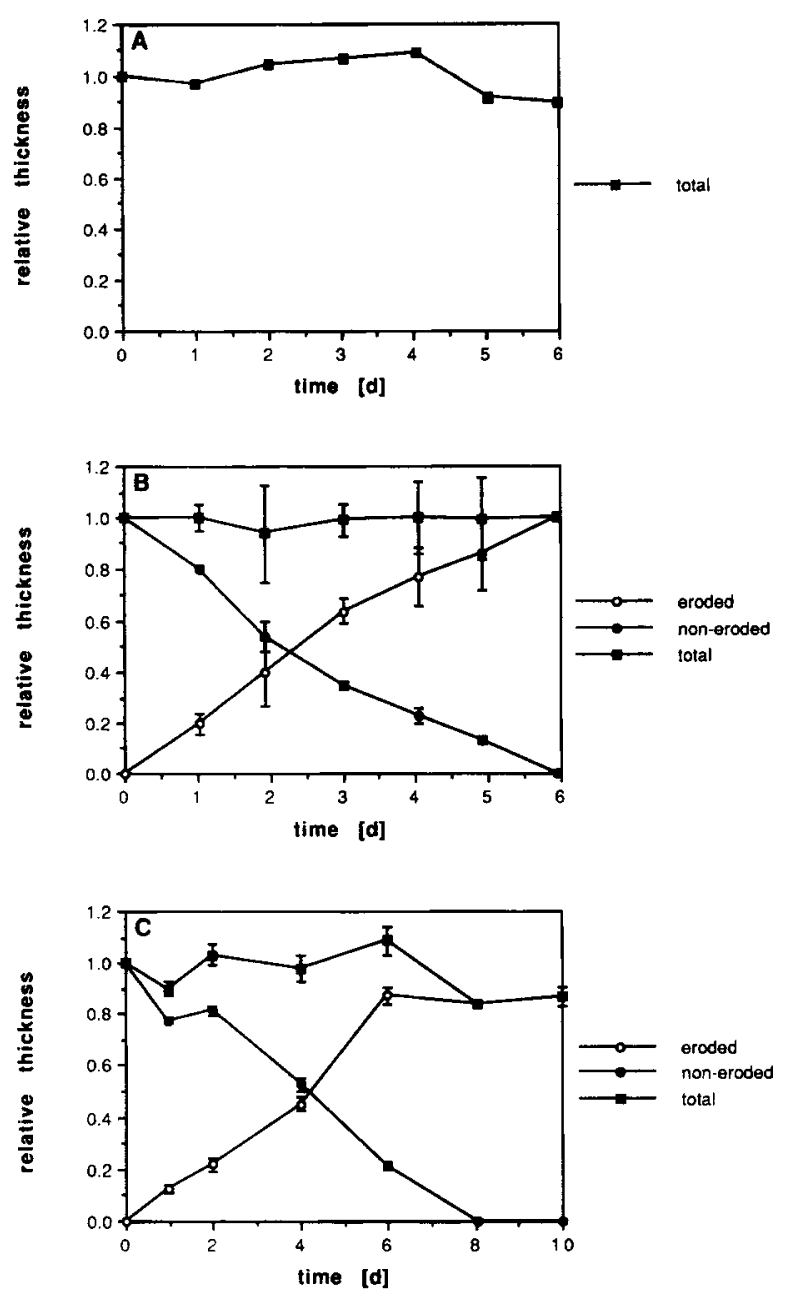

Figure 14. Change of the thickness of the eroded-, the noneroded zone and of their sum during erosion relative to the thickness at $t=0:$ (A) $p$ (SA), (B) $p$ (CPP-SA) $20: 80$, (C) $p$ (CPP-SA) $50: 50$.

investigate the change of weight during erosion because this would allow estimation of total porosity. The change of matrix weight during erosion is shown in Figure 15 (a). There are some common features for all three polymers. During the initial stage of erosion the velocity of weight loss is relatively slow. After 1 day all three polymers enter a phase of nearly constant weight loss indicating that the system has reached a steady state. This lasts for a couple of days after which the mass loss declines which indicates that processes other than only chain scission of the polymer become important. The results are in good agreement with results for other polyanhydrides. ${ }^{22}$

The change in porosity during erosion was investigated by mercury porosimetry. The porosity was calculated from the ratio of the volume of intruded mercury and total disc volume. Additionally a theo- 

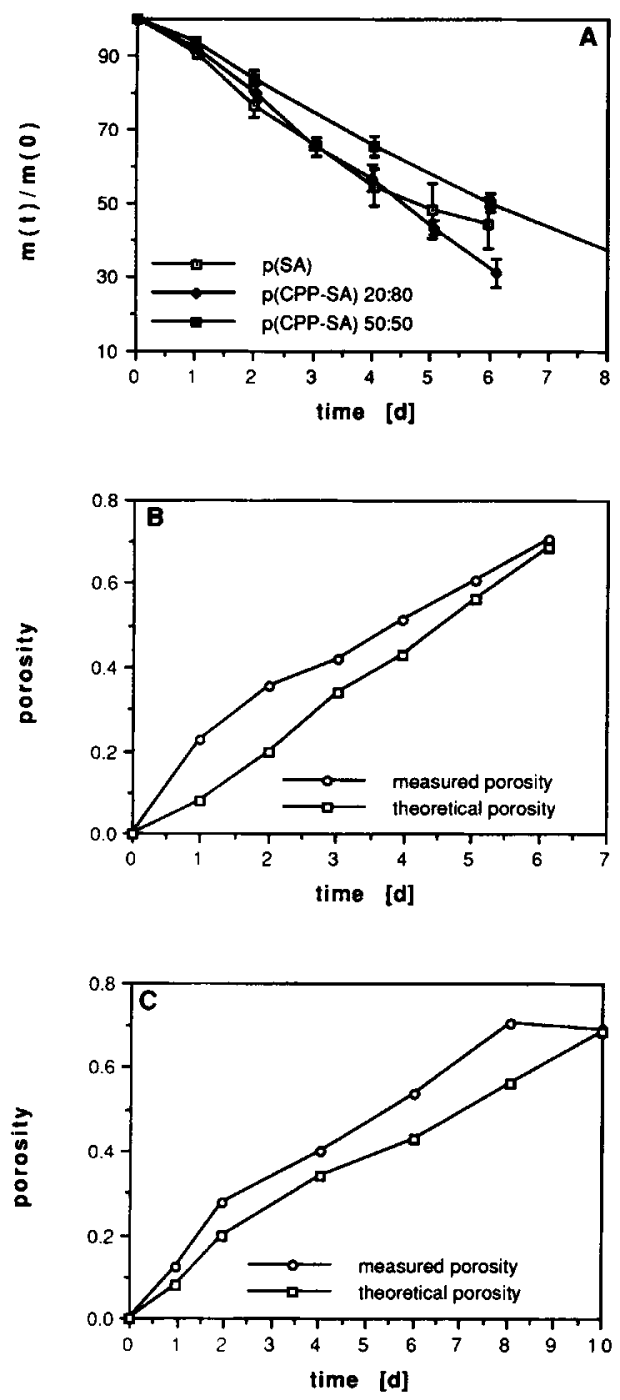

Figure 15. Relative weight and porosity of polymer discs during erosion: (A) relative weight of all polymers during erosion, (B) theoretical and measured porosity of $p$ (SA), (C) theoretical and measured porosity of $p$ (CPPSA) $20: 80$.

retical value for the porosity was calculated from the ratio of the weight after degradation to the disc volume. Figures 15(b) and (c) show the measured porosity as a function of time and the theoretical porosity calculated from the weight loss for the two copolymers. $p(\mathrm{SA})$ was too brittle to yield undamaged discs and could not be measured. The measured porosity is higher in both cases than the porosity estimated from weight loss. This confirms the changes in crystallinity during erosion which were seen by DSC. $p$ (CPP-SA) $20: 80$ erodes heterogeneously with respect to amorphous and crystalline regions (Fig. 4). Amorphous regions have a lower density but erode faster than crystalline regions.
Together with crystallization of monomers this increases the density of the polymer discs and the loss of weight is no longer proportional to porosity. In the case of $p$ (CPP-SA) $50: 50$ there are no substantial crystalline areas and the changes in density are caused only by crystallization of monomers. The pore sizes estimated from the Washburn equation shift to higher values with time. They are initially at a submicron level which is in good agreement with the findings from SEM. A more precise determination of pore size diameters is not possible as the contact angle between mercury and the polymer changes with advancing erosion due to chemical changes inside the polymers.

\section{The Release of Monomers during Erosion}

A method which has been used extensively by many research groups to follow the erosion of polymers is the determination of monomer release with time. The release of monomers may depend on many factors such as device geometry, porosity, and monomer solubilities. It is therefore not easy to explain all the features of the release profiles. On the basis of results in the previous sections we are, however, able to explain the release of monomers from these polymers in more detail than was previously possible.

Figures 16(a)-(c) show the relative release of monomers for the three polymers during erosion determined by HPLC. The release of SA from $p$-SA in Figure 16(a) is similar to the release from monolithic devices containing suspended drug. ${ }^{23} \mathrm{~A}$ lag period during the first hours of erosion is visible which was also observed for the loss of weight [Fig. 15(a)]. The lag-phase is followed by a short period of linear release. After 3 days the release velocity slows down which coincides with the time after which there is no more crystalline SA present [Fig. 13(a)]. At that point most of the amorphous parts of the device have eroded [Fig. 12(a)]. The release of monomers continues for more than 30 days. From DSC studies it can be assumed that the monomers released at that time come from the crystalline skeleton of eroded spherulites.

Figures 16(b) and (c) show that $p$ (CPP-SA) $20: 80$ and $p$ (CPP-SA) $50: 50$ release the monomers in a similar way to each other but different from the homopolymer. The initial lag-period of erosion is again visible. In contrast to the homopolymer the release of SA from the copolymers is faster. Between day 1 and 6 the release is almost constant. After ca. 7 days all SA has been released from both copolymers which coincides with the disappearance of crystalline SA from the devices seen by DSC [Figs. 

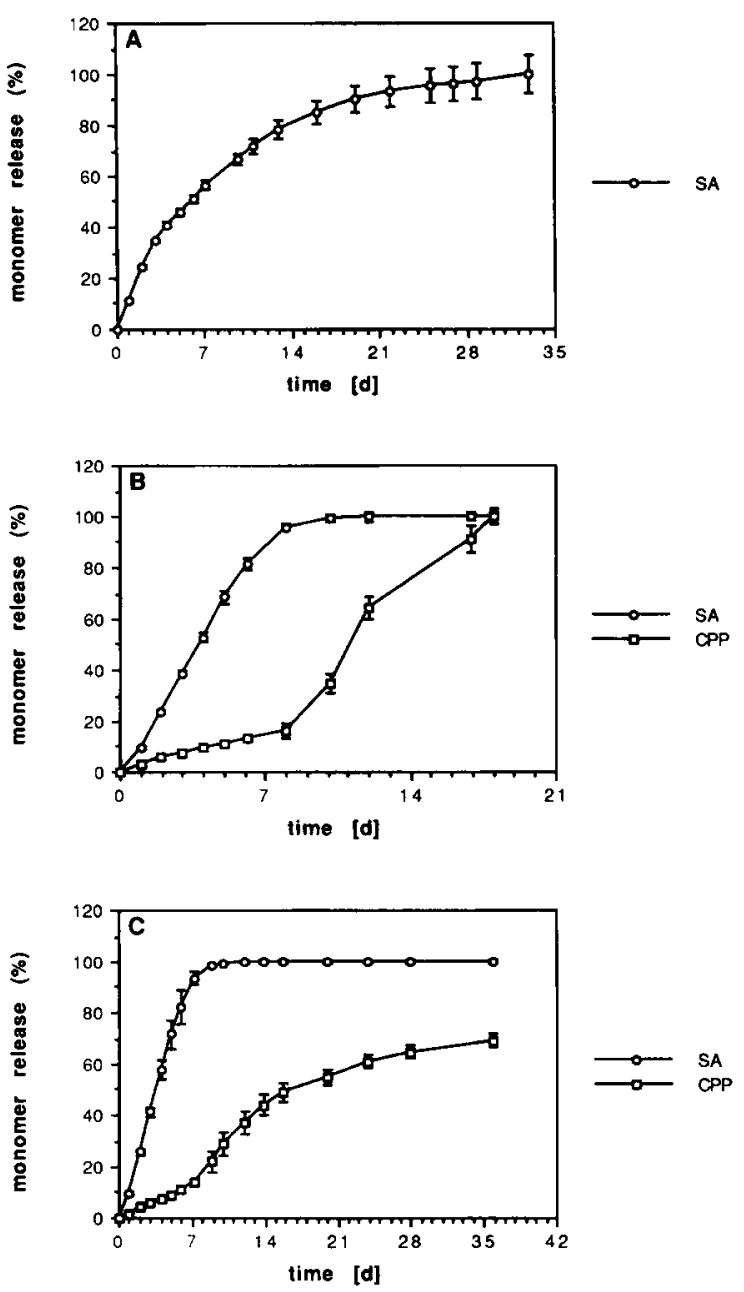

Figure 16. Release profiles of CPP and SA monomer during erosion: (A) $p$ (SA), (B) p (CPP-SA) $20: 80$, (C) $p$ (CPP-SA) $50: 50$.

13(b) and (c)]. The identical release of SA from both polymers is also in agreement with the movement of their erosion front [Figs. 14(b) and (c)], which reaches the center of the device at the same time. Compared to SA the release of CPP is much slower and more complicated. During an initial period of ca. 7 days the release of CPP is almost linear. It then increases instantaneously displaying a slightly sigmoid profile. The release is triggered by the disappearance of SA. This discontinuity can be explained by the solubility of the monomers (Fig. 5). It was found that both substances have a similar first $\mathrm{p} K_{a}$ value but that $\mathrm{SA}$ is five times more soluble than CPP. The $\mathrm{pH}$ will, therefore, be determined mainly by SA. Whenever SA has left the device, the pH will rise and CPP will become more soluble. As the release rate depends on the solubility ${ }^{23}$ it will increase. Two major factors influence monomer release rates and will cause the slower release rate of CPP compared to that of SA. First, because of CPP's higher molecular weight relative to that of SA we can conclude from the Stokes-Einstein equation that CPP will diffuse more slowly through the highly porous and tortuous eroded zone. Second, the lower solubility of CPP relative to $\mathrm{SA}$ affects its release rate. After 1 week the low dissolution velocity of crystalline CPP seems to be the major reason for the low release rate of this monomer.

\section{CONCLUSIONS}

The two major factors that determine the erosion of the investigated polyanhydrides are their microstructure and the chemical properties of the monomers.

By SEM it could be seen that the crystalline skeleton of the polymers degrades slower than their amorphous parts. The created erosion zones are characterized by a connected porous network in which the mean pore diameter is ca. $0.1-2 \mu \mathrm{m}$. From $\mathrm{pH}$ measurements in the buffer solution and on the surface of the devices by scanning confocal microscopy it could be concluded that the $\mathrm{pH}$ inside the pores is determined by the dissolved monomers. By wide-angle $\mathrm{x}$-ray spectroscopy and DSC it was found that the monomers crystallize during erosion inside the porous network which explains the almost constant release of the monomers [Figs. 16(a)-(c)]. The release rate of CPP increases, however, spontaneously when SA has been completely released. The reason is the change of $\mathrm{pH}$ inside the devices. In a saturated solution of both monomers the $\mathrm{pH}$ can be approximated by the following simplified equation: ${ }^{24}$

$$
\mathrm{pH}=\frac{1}{2} \log \left(c_{\mathrm{SA}} K_{a_{\mathrm{SA}}}+c_{\mathrm{CPP}} K_{a_{\mathrm{CPP}}}\right)
$$

where $c$ denotes the solubility of CPP or SA. As the solubility of SA is at least five times higher with nearly identical $\mathrm{p} K_{a}$ values, the $\mathrm{pH}$ is determined by this monomer. Whenever SA has left the device the $\mathrm{pH}$ and, thereby, the CPP solubility will rise, resulting in a faster release.

The composition of the devices changes greatly during erosion. $p(\mathrm{SA})$ erodes to a highly porous highly crystalline network. The crystalline regions in $p$ (CPP-SA) $20: 80$ are much more liable to deg- 
radation due to the hindered regular arrangement of the polymer chains. There seem to be some polymer remains ${ }^{11}$ but for the most part the erosion zone consists of crystallized CPP monomer. In the case of $p$ (CPP-SA) $50: 50$ there is even more crystallized CPP. After ca. 10 days of erosion the copolymers consist mainly of crystallized CPP monomer that dissolves very slowly besides slowly degrading CPP rich oligomers. ${ }^{22}$

Special thanks are to the National Science Foundation and to the Deutsche Forschungsgemeinschaft, Bonn, Germany, which funded this project by Grants CTS 8918587, the National Cancer Institute, CA 52857, and GO 565/1-1. The authors acknowledge Nova Pharmaceuticals, Baltimore, MD, which provided the monomers and polymers for this study. Special thanks are also due to Manoj Maniar and Michael Adams who synthesized the polymers.

\section{REFERENCES AND NOTES}

1. S. Stupp and G. Ciegler, J. Biomed. Mat. Res., 26, 169-183 (1992).

2. L. G. Cima, J. P. Vcanti, C. Vacanti, D. Ingber, D. Mooney, and R. Langer, J. Biomech. Eng., 113, 143151 (1991).

3. R. W. Baker, Controlled Release of Biologically Active Agents, Wiley, New York, 1987, pp. 84-131.

4. A. Joshi and K. J. Himmelstein, J. Contr. Rel., 15, 95-104 (1991).

5. S. M. Li, H. Garreau, and M. Vert, J. Mat. Sci. Mat. Med., 1, 131-139 (1990).

6. K. W. Leong, B. C. Brott, and R. Langer, J. Biomed. Mater. Res., 19, 941-955 (1985).

7. S. M. Li, H. Garreau, and M. Vert, J. Mat. Sci. Mat. Med., 1, 123-130 (1990).

8. M. Vert, S. Li, and H. Garreau, J. Contr. Rel., 16, 15-26 (1991).
9. J. Tamada and R. Langer, J. Biomater. Sci. Polym. Ed., 3(4), 315-353 (1992).

10. E. Mathiowitz, E. Ron, G. Mathiowitz, C. Amato, and R. Langer, Macromolecules, 23, 3212-3218 (1990).

11. J. Tamada and R. Langer, Proc. Nat. Acad. Sci., 90, 552-556 (1993).

12. A. Domb, E. Ron, and R. Langer, Polyanhydrides: Encyclopedia of Polymer Science and Engineering, supplement volume, 2nd ed., Wiley, New York, 1989, pp. 648-665.

13. P. C. Hiemenz, Polymer Chemistry, Dekker, New York and Basel, 1984, pp. 235-244.

14. D. C. Bassett, Principles of Polymer Morphology, Cambridge University Press, Cambridge, 1981, pp. 2228.

15. F. W. Billmeyer, Textbook of Polymer Science, 3rd ed., Wiley, New York, 1984, pp. 273-281.

16. J. M. Schultz, Polymer Material Science, PrenticeHall, Englewood Cliffs, NJ, 1974, pp. 154-164.

17. C. Laurencin, Ph.D. thesis, 1987.

18. J. Tamada and R. Langer, Proc. Int. Symp. Control. Rel. Bioact. Mater., 17, D305 (1990).

19. R. Langer and N. Peppas, J. Macromol. Sci. Rev. Macromol. Chem. Phys., C23, 61-126 (1983).

20. G. Engeln-Muellges and F. Reuter, Formelsammlung zur numerischen Mathematik mit Standard-Fortran 77 Programmen, 5th ed., Bibliographisches Institut Mannheim, Wien, Zürich, 1986, pp. 162-164.

21. P. Sykes, A Guidebook to Mechanism in Organic Chemistry, 4th ed., Longman Group, London, 1975, pp. 52-76.

22. A.-C. Albertson and S. Lundmark, Br. Polym. J., 23, 205-212 (1990).

23. A. Göpferich and G. Lee, Drug Dev. Ind. Pharm., 18(3), 319-331 (1992).

24. U. Kunze, Grundlagen der quantitativen Analyse, 1st ed., Georg Thieme Verlag, Stuttgart, New York, 1980, p. 57.

Received September 16, 1992

Accepted February 8, 1993 